Utah State University

DigitalCommons@USU

\title{
Properties of Cathodoluminescence for Cryogenic Applications of SiO2-based Space Observatory Optics and Coatings
}

\author{
Amberly Evans Jensen \\ Utah State University \\ JR Dennison \\ Utah State Univesity \\ Gregory Wilson \\ Utah State University \\ Justin Dekany \\ Charles Bpwers \\ NASA Goddard Space Flight Center \\ Robert Meloy \\ MEI Technologies, Inc. \\ Follow this and additional works at: https://digitalcommons.usu.edu/mp_conf \\ Part of the Physics Commons \\ See next page for additional authors
}

\section{Recommended Citation}

Amberly Evans Jensen, JR Dennison, Gregory Wilson, Justin Dekany, Charles W. Bowers, Robert Meloy and James B. Heaney, Properties of Cathodoluminescence for Cryogenic Applications of SiO2-based Space Observatory Optics and Coatings," Proceedings of the Society of Photo-Optical Instrumentation Engineers Cryogenic Optical Systems and Instruments Conference, Vol. 8863, 2013, pp.

88630A1-88630A10. DOI: 10.1117/12.2030231

This Article is brought to you for free and open access by the Materials Physics at DigitalCommons@USU. It has been accepted for inclusion in Conference Proceedings by an authorized administrator of DigitalCommons@USU. For more information, please contact digitalcommons@usu.edu.

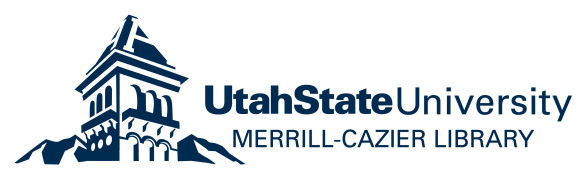


Authors

Amberly Evans Jensen, JR Dennison, Gregory Wilson, Justin Dekany, Charles Bpwers, Robert Meloy, and James B. Heaney 


\title{
Properties of Cathodoluminescence for Cryogenic Applications of $\mathrm{SiO}_{2}$-based Space Observatory Optics and Coatings
}

\author{
Amberly Evans Jensen ${ }^{* a}$, JR Dennison ${ }^{\mathrm{a}}$, Gregory Wilson ${ }^{\mathrm{a}}$, Justin Dekany ${ }^{\mathrm{a}}$, \\ Charles W. Bowers ${ }^{\mathrm{b}}$, Robert Meloy ${ }^{\mathrm{c}}$, and James B. Heaney ${ }^{\mathrm{d}}$ \\ ${ }^{a}$ Materials Physics Group, Physics Department, Utah State University \\ ${ }^{\mathrm{b}}$ NASA Goddard Space Flight Center, ${ }^{\mathrm{c}}$ MEI Technologies, Inc. \\ ${ }^{\mathrm{d}}$ Stinger Ghaffarian Technologies, Inc.
}

\begin{abstract}
Disordered thin film $\mathrm{SiO}_{2} / \mathrm{SiO}_{\mathrm{x}}$ coatings undergoing electron-beam bombardment exhibit cathodoluminescence, which can produce deleterious stray background light in cryogenic space-based astronomical observatories exposed to highenergy electron fluxes from space plasmas. As future observatory missions push the envelope into more extreme environments and more complex and sensitive detection, a fundamental understanding of the dependencies of this cathodoluminescence becomes critical to meet performance objectives of these advanced space-based observatories. Measurements of absolute radiance and emission spectra as functions of incident electron energy, flux, and power typical of space environments are presented for thin $(\sim 60-200 \mathrm{~nm}) \mathrm{SiO}_{2} / \mathrm{SiO}_{\mathrm{x}}$ optical coatings on reflective metal substrates over a range of sample temperatures $(\sim 40-400 \mathrm{~K})$ and emission wavelengths $(\sim 260-5000 \mathrm{~nm})$. Luminescent intensity and peak wavelengths of four distinct bands were observed in UV/VIS/NIR emission spectra, ranging from 300 $\mathrm{nm}$ to $1000 \mathrm{~nm}$. A simple model is proposed that describes the dependence of cathodoluminescence on irradiation time, incident flux and energy, sample thickness, and temperature.
\end{abstract}

Keywords: space environment, light emission, cathodoluminescence, optical coatings, cryogenic, stray light, spacebased observatories

\section{INTRODUCTION}

Recent and planned space-based astronomical observatory missions push the design and performance envelope, as these missions use more complex and sensitive detectors, reside in more extreme and remote environments, become larger and require open architectures, and operate at broader temperature ranges. For example, to meet its demanding science objectives, the ambitious James Webb Space Telescope (JWST) space-based observatory project currently being constructed by NASA is designed for very high sensitivity over a $0.6 \mu \mathrm{m}$ to $27 \mu \mathrm{m}$ bandpass, resides in the dynamic L2 environment, has an open architecture with very large collecting area $\left(\geq 25 \mathrm{~m}^{2}\right)$, and will operate at cryogenic temperatures $\lesssim 40 \mathrm{~K}^{1}$. These extended operational requirements for space-based observatories, either individually or synergistically, can raise new issues for optics elements and coatings subject to space environment effects. One such concern is electron-induced luminescence (or cathodoluminescence) of optical and coatings exposed to high-energy electron fluxes from space plasmas ${ }^{2}$. Such emission from optical components can produce an additional background source as light propagates to instrument focal planes, limiting the observatory sensitivity. This study characterizes the phenomena of cathodoluminescence for a specific, ubiquitous class of optical coatings- disordered $\mathrm{SiO}_{2} / \mathrm{SiO}_{\mathrm{x}}$ thin films, frequently used as protective overcoatings for highly reflective mirrors — and provides estimates of the effects of space environment parameters such as electron flux and energy spectrum, coating temperature, and film thickness on the absolute spectral radiance of the electron-induced luminescence. A fundamental understanding of the dependencies of cathodoluminescent intensity on these parameters combined with the environmental electron spectrum and the sensitivity of the observatory to such optical glow, allows an assessment of the impact to observatory performance.

*amb.eva@aggiemail.usu.edu; phone 435-363-4704

Cryogenic Optical Systems and Instruments 2013, edited by James B. Heaney,

E. Todd Kvamme, Proc. of SPIE Vol. 8863, 88630A - (c) 2013 SPIE

CCC code: $0277-786 \mathrm{X} / 13 / \$ 18 \cdot$ doi: $10.1117 / 12.2030231$ 


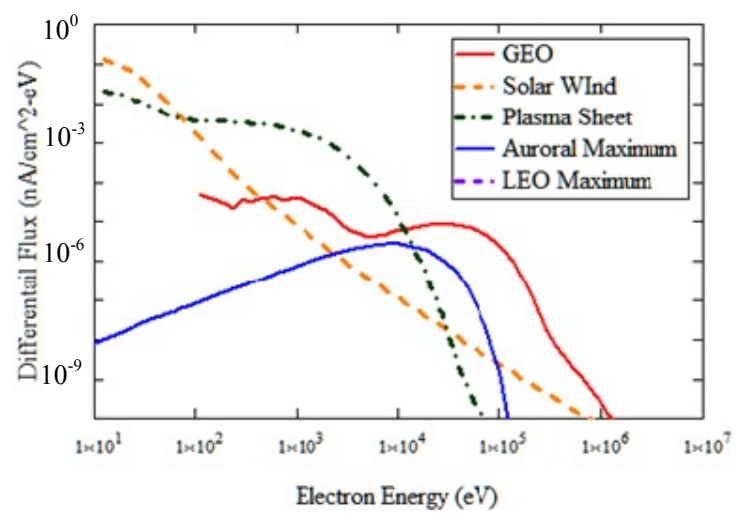

(a)

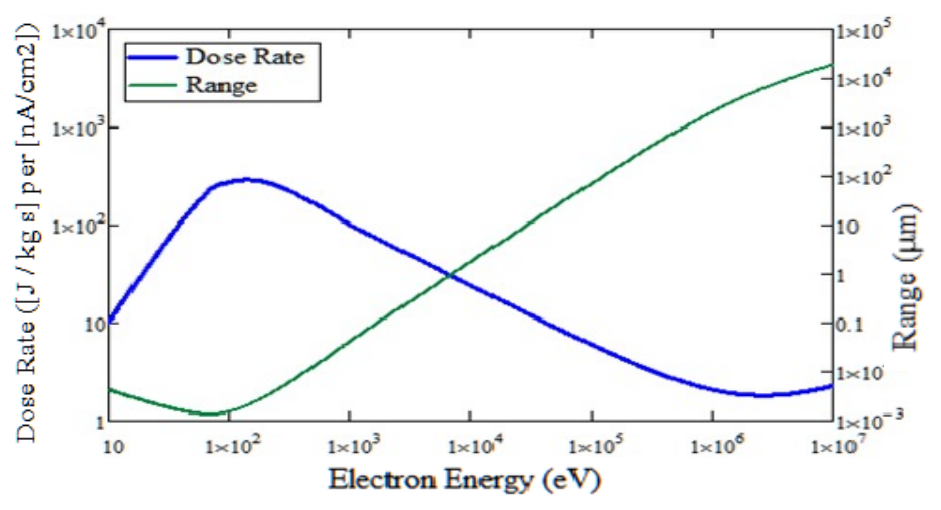

(b)

Figure 1. (a) Typical space differential electron flux spectra for geostationary earth orbit, ${ }^{3,4}$ solar wind at the mean earth orbital distance ${ }^{5}$, Earth plasma sheet ${ }^{6}$, a peak auroral storm $^{3}$, and a maximum low earth (ISS) orbit ${ }^{3}$. (b) Range and dose rate of disordered $\mathrm{SiO}_{2}$ as a function of incident energy using calculation methods and the continuous slow-down approximation described by Wilson, et al. ${ }^{7}$.

The space plasma environment creates a harsh setting in which spacecraft reside. One important consequence of space plasma interactions that accounts for the majority of spacecraft anomalies produced by the space environment ${ }^{8}$ is spacecraft charging; this is the accumulation of an electrical charge on orbiting spacecraft induced by the space plasma environment and can have harmful effects on the electrical functionality of a spacecraft ${ }^{3}$. However, the magnitude of cathodoluminescence will also be significantly affected by the environmental conditions, in particular the energy spectrum of impacting electrons. Energy spectra typical of five common space plasma environments (GEO, LEO, auroral, plasma sheet and solar wind), dominated by fluxes $\left(\sim 0.01 \mathrm{pA} / \mathrm{cm}^{2}\right.$ to $\left.\sim 10 \mathrm{nA} / \mathrm{cm}^{2}\right)$ for electron energies of up to $\sim 100 \mathrm{keV}^{3}$, are shown in Fig. 1(a). Environmental electron fluxes often show a wide variance due to storm conditions, stochastic variability and changes due to the different environments which may be encountered during a particular mission; the curves in Fig. 1(a) are intended to illustrate the range of some of these different environments ${ }^{3-6}$. Severe space weather conditions for environments such as those illustrated in Fig. 1(a) have on the order of $10 \mu \mathrm{W}-\mathrm{cm}^{-2}$ incident electron power (i.e., $10 \mathrm{nA}-\mathrm{cm}^{-2}$ and $1 \mathrm{keV}$ or $1 \mathrm{nA}-\mathrm{cm}^{-2}$ and $10 \mathrm{keV}$ ); this provides a scaling factor for comparison of luminescent intensities observed in the experiments describe below.

Previous studies have observed cathodoluminescence in both epoxy resin composites ${ }^{9}$ and in disordered $\mathrm{SiO}_{2}{ }^{10}$ at room temperature. In addition, recent charging studies at Utah State University (USU) using low-flux intermediate-energy electron beams on thin film highly disordered $\mathrm{SiO}_{2}$ samples exhibited not only cathodoluminescent behavior ${ }^{3,11-14}$ but also occasional arcing under some conditions. Similar behavior was also observed in experiments for other dielectric materials including cyanate ester and epoxy resins in graphite and fiberglass composites ${ }^{2,9}$ and Kapton $\mathrm{HN}^{\mathrm{TM}}$ polyimide and carbon-loaded polyimide nanodielectric composites ${ }^{2,13}$. These results suggest that materials used in structural components, optical elements, and thermal control surfaces of spacecraft and space-based observatories could, when exposed to the space plasma environment, luminesce. However little information is available with regard to the temperature dependence of the cathodoluminescence. Because IR instrumentation is temperatuere sensitive and NASA's IR observatories are positioned in space accordingly, it is imperative to know and understand the material luminescent behavior at the operating temperature, rather than simply at room temperature. This motivated a study of the dependence of the cathodoluminescent intensity of $\mathrm{SiO}_{2}$ optical coatings on incident electron flux, energy and power and on material temperature and thickness. Measurements for fluxes of well-characterized, monoenergetic electron beams are presented of the absolute radiance and emission spectra as functions of incident electron energy, flux and power for vapor deposited silicon dioxide coatings ( $\sim 60 \mathrm{~nm}$ to $\sim 200 \mathrm{~nm}$ thick) on reflective metal substrates over a range of sample temperatures ( $\sim 40 \mathrm{~K}$ to $\sim 400 \mathrm{~K})$ and emission wavelengths $(\sim 250 \mathrm{~nm}$ to $\sim 1900 \mathrm{~nm})$.

\section{EXPERIMENTATION}

Our experiments measured the electrical and optical response of sample materials $\left(\sim 1-5 \mathrm{~cm}^{2}\right.$ area) bombarded with a beam of electrons. These were conducted in the main USU electron emission ultrahigh vacuum test chamber ${ }^{15}$, modified 


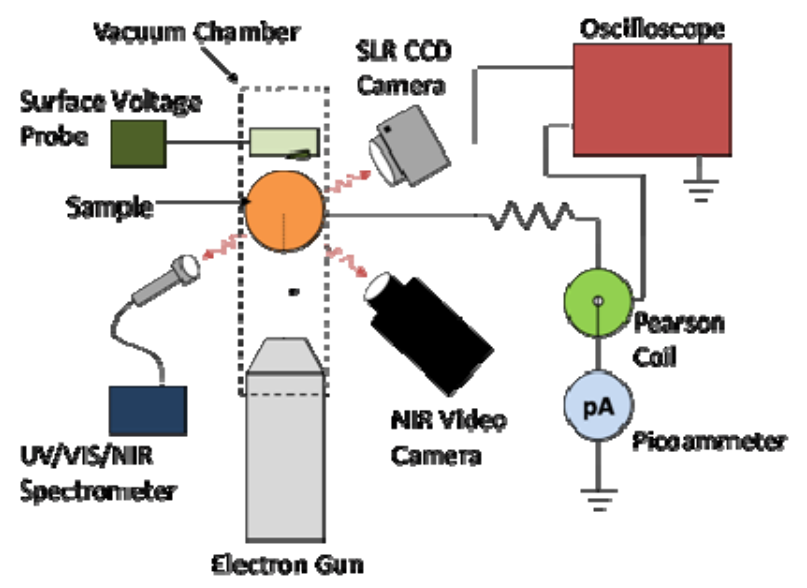

(a)

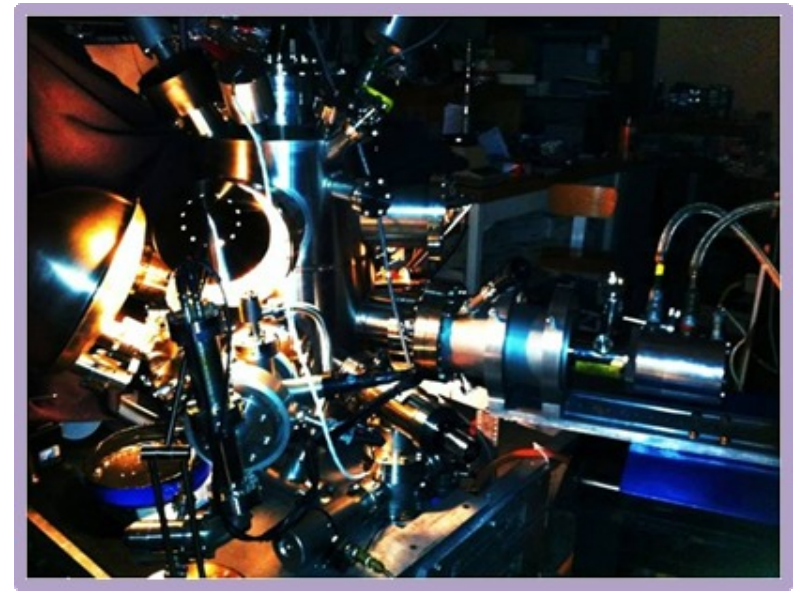

(b)

Figure 2. (a) Block diagram of instrumentation for collecting the pulse charging surface voltage, electrode current and cathodoluminescence data induced by electron beam bombardment. Instrumentation includes picoammeters, Pearson coils, and a storage oscilloscope for electrode current measurements and UV/VIS and IR spectrometers, an SLR CCD still camera, and a NIR video camera for optical measurements. (b) Photograph of instrumentation mounted to USU Electron Emission Test Chamber.

Table 1. Camera Sensitivity and Background Radiance

\begin{tabular}{|l|c|c|c|c|c|}
\hline Camera & $\begin{array}{c}\text { Bandpass } \\
(\mathrm{nm})\end{array}$ & $\begin{array}{c}\text { Weighted Central } \\
\text { Wavelength } \\
(\mathrm{nm})\end{array}$ & $\begin{array}{c}\text { Band- } \\
\text { width } \\
(\mathrm{nm})\end{array}$ & $\begin{array}{c}\text { Detection Threshold } \\
\text { Spectral Radiance }^{\mathrm{a}} \\
{\left[\mathrm{W}-(\mathrm{cm}-\mathrm{nm}-\mathrm{sr})^{-1}\right]}\end{array}$ & $\begin{array}{c}\text { Zodiacal Background } \\
\text { Spectral Radiance }^{\mathrm{b}} \\
{\left[\mathrm{W}-(\mathrm{cm}-\mathrm{nm}-\mathrm{sr})^{-1}\right]}\end{array}$ \\
\hline SLR CCD Camera & $390-650$ & 553 & 260 & $4 \cdot 10^{-15}$ & $7 \cdot 10^{-14}$ \\
\hline CCD Video Camera & $400-900$ & 830 & 500 & $4 \cdot 10^{-16}$ & $3 \cdot 10^{-14}$ \\
\hline InGaAs Video Camera & $950-1700$ & 1512 & 750 & $8 \cdot 10^{-16}$ & $1 \cdot 10^{-14}$ \\
\hline
\end{tabular}

a Assumes linear power scaling at an incident electron power of $10 \mu \mathrm{W}-\mathrm{cm}^{-2}$.

${ }^{b}$ Assumes broadband continuum emissions evenly distributed across the camera bandwidths for a zodiacal sky background at 1AU near the south ecliptic pole for the weighted central wavelength ${ }^{16,17}$.

for observations of low intensity UV/VIS/NIR glow over a $40 \mathrm{~K}$ to $400 \mathrm{~K}$ range of sample temperatures ${ }^{18}$. Figure 2 provides a schematic and image of the experimental system used. Details of the experimental set up, electron sources and beam characterization, optical detectors, methods, calibration, and the sample mounting carousel and thermal stage, are given by Dennison et al. ${ }^{15}$. Additional details of the thermal dependence of the cathodoluminescence are presented by Jensen et al. ${ }^{14}$. Electron transport, surface voltage data, and electrostatic discharge measurements acquired simultaneously with luminescence data are reported by Wilson et al. ${ }^{11}$.

Electrons were directed to the samples using well-characterized, low-flux electron beams $\left(\sim 50 \mathrm{pA} / \mathrm{cm}^{2}\right.$ to $\left.1 \mu \mathrm{A} / \mathrm{cm}^{2}\right)$ with an energy range of 5-25 keV. For most results shown in Figs. 3 and 4, beams of $5 \mathrm{keV}$ at $\sim 500 \mathrm{nA} / \mathrm{cm}^{2}$ from a low energy electron gun [Staib, EK-5-51;20 eV to $5 \mathrm{keV}$ ] were used. The defocused electron beam produced a beam profile at the sample with about $\pm 30 \%$ uniformity over an $\sim 3 \mathrm{~cm}$ diameter beam spot. Beam fluxes were measured before and after each experiment with a Faraday cup and were stable to within $\pm 5 \%$. The $\mathrm{W}$ filament of the electron gun also produced visible light that traveled down the bore of the electron gun and impinged on the sample; even at the low operating filament temperatures used for the low flux beam $(\sim 2500 \mathrm{~K})$, the intensity of the filament spot on the sample was initially larger than the luminescent signals. To minimize this background filament light, a blocking aperture was developed, which allowed an electron beam focused $\sim 5^{\circ}$ off axis and then electrostatically deflected back on to the sample while sending the filament light into an optical baffle. The baffle and the primarily specular nature of the reflected filament light that made it past the blocking aperture reduced the stray light background seen by the optical cameras to acceptable levels. 


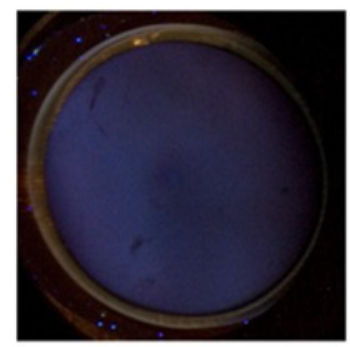

(a)

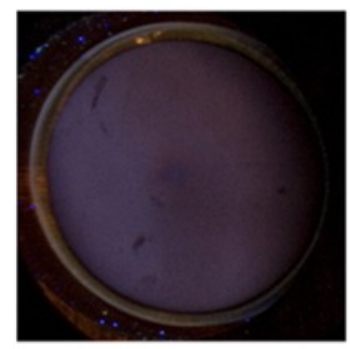

(c)

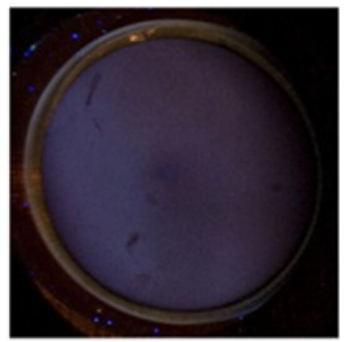

(b)

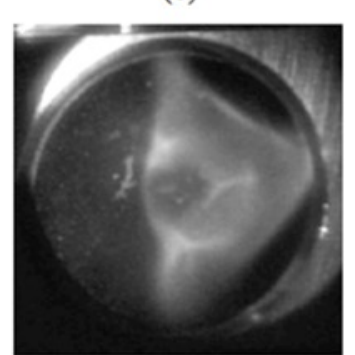

(d)

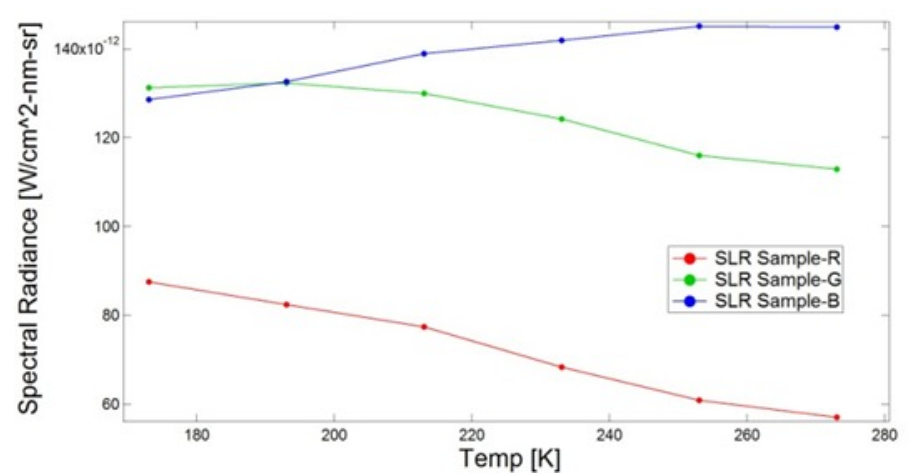

(e)

Figure 3. (Left) Optical images of luminescent $\mathrm{SiO}_{2}$ samples. SLR still images of the glowing thin film $\mathrm{SiO}_{2}$ sample at (a) $273 \mathrm{~K}$, (b) $193 \mathrm{~K}$ and (c) $163 \mathrm{~K}$. Note that the lower temperature image has a very red tint to it. However, as the temperature continues to rise, and eventually reaches room temperature, the color begins to shift to blue. (d) CCD video camera frame showing the luminosity produced by a highly distorted, defocused electron beam. (e) Variation with sample temperature of the Red-Green-Blue components of the SLR still CCD camera image average spectral radiances across the glowing thin film disordered $\mathrm{SiO}_{2}$ sample.

Two cameras and two fiber optic spectrometers were used to monitor very low light intensity (see Table 1). Optical spectra were collected using UV/VIS (Stellarnet, 13LK-C-SR; $\sim 200 \mathrm{~nm}$ to $1080 \mathrm{~nm}$ with $\sim 1 \mathrm{~nm}$ resolution) and NIR (Stellarnet, RW-InGaAs-512; $\sim 1000 \mathrm{~nm}$ to $1700 \mathrm{~nm}$ with $\sim 3 \mathrm{~nm}$ resolution) spectrometers. Imagery of the electron illuminated sample was obtained with an SLR CCD still camera (Cannon, EOS Rebel XT DS126071; 390 nm to 650 $\mathrm{nm}, 30 \mathrm{~s}$ per frame), a VIS/NIR image-intensified CCD video camera (Xybion, ISG-780-U-3; 400 nm to $900 \mathrm{~nm}, 30$ frames per s), and InGaAs (Goodrich Sensors Unlimited, SU320MS-1.7RT; $\sim 800 \mathrm{~nm}$ to $1700 \mathrm{~nm}, 60$ frames/s) and InSb $(\sim 1000 \mathrm{~nm}$ to $5500 \mathrm{~nm}$; Santa Barbara Focalplane, SBF119) video cameras. Various filter combinations were used to measure IR emissions; no IR in the $900 \mathrm{~nm}$ to $2500 \mathrm{~nm}$ range was observed for any of the experiments reported here. For each instrument the spectral response and wavelength range were determined and the sensitivity was calibrated with NIST traceable sources prior to the luminescence studies (see Table 1) ${ }^{18}$. Visible range calibrations for the SLR CCD camera, CCD video camera and InGaAs video camera were done using a 6 in diameter Spectralon ${ }^{\mathrm{TM}}$ integrating sphere (Labsphere, Model SC6000) with a NIST traceable calibration curve for absolute spectral radiance, using an integrated W-halogen lamp powered by a NIST traceable calibrated current source (Keithley, Model 6430). A smaller 1.5 in diameter Spectralon ${ }^{\mathrm{TM}}$-lined fiber optic integrating sphere (Ocean Optics, Model FOIS-1) connected to a calibrated Whalogen blackbody $(2530 \mathrm{~K})$ source (Ocean Optics, Model LS-1-CAL-INT) was used as an in vacuo secondary standard. NIR calibrations for the InGaAs and InSb video cameras were done in vacuo using a NIST traceable blackbody calibration source (CI Systems, Model SR80) operating at $273 \mathrm{~K}$ to $373 \mathrm{~K}$ with $\pm 25 \mathrm{mK}$ stability.

The samples ( $2.5 \mathrm{~cm}$ diameter) studied here had an optically smooth, disordered $\mathrm{SiO}_{2}$ thin optical coating $(\sim 65 \mathrm{~nm}$ thick) deposited on $\sim 175 \mathrm{~nm}$ thick highly reflective, optically smooth metal (mostly $\mathrm{Au}$ ) layers on a $2 \mathrm{~mm}$ thick fused quartz substrate. The samples were optically cleaned and underwent a $\sim 12 \mathrm{hr}$ vacuum bakeout at $\sim 390 \mathrm{~K}$ and $<1 \bullet 10^{-3} \mathrm{~Pa}$ to eliminate adsorbed water and volatile contaminants. The samples were mounted on $\mathrm{Cu}$ pedestals on a multi-sample carousel, and were placed in an ultrahigh vacuum chamber (base pressure $<1 \bullet 10^{-6} \mathrm{~Pa}$ ) for $>24 \mathrm{hrs}$ to allow outgassing before measurements were made. The sample carousel was thermally anchored to (but electrically isolated from) a thermal reservoir. In combination with resistive heaters and liquid $\mathrm{N}_{2}$ cryogen, the samples were maintained over a range of temperatures from $\sim 150 \mathrm{~K}$ to $\sim 400 \mathrm{~K}$ with a stability of $\pm 4 \mathrm{~K}$ maintained over typical $2 \mathrm{hr}$ experiment durations. Lower temperature data were acquired using a closed-cycle He refrigerator based system from $\sim 40 \mathrm{~K}$ to $\sim 300 \mathrm{~K}$ with a 


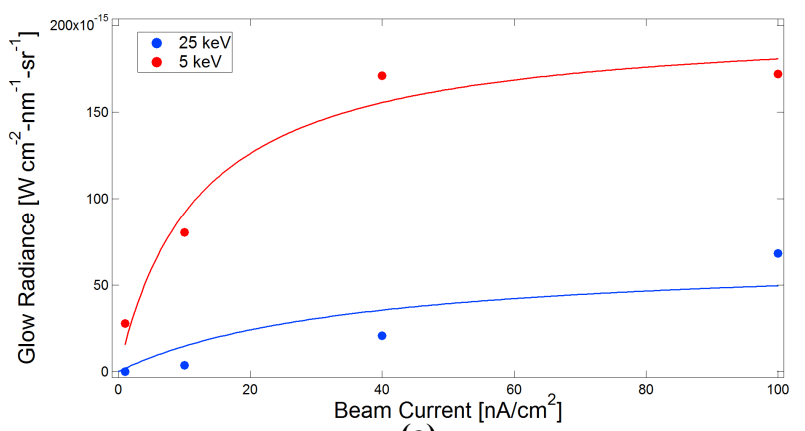

(a)

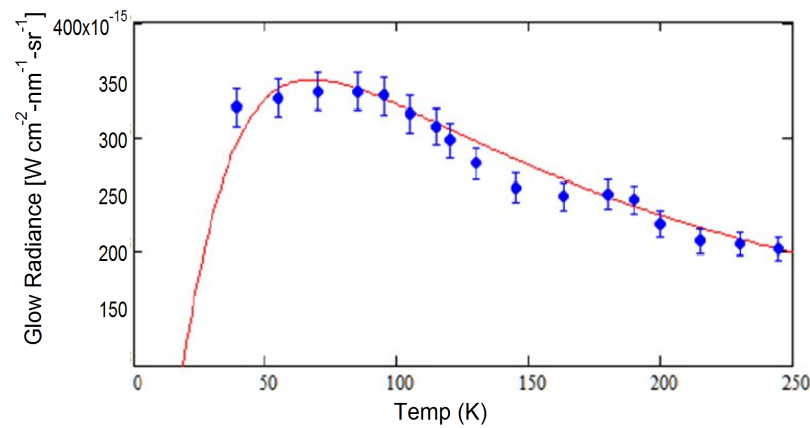

(c)

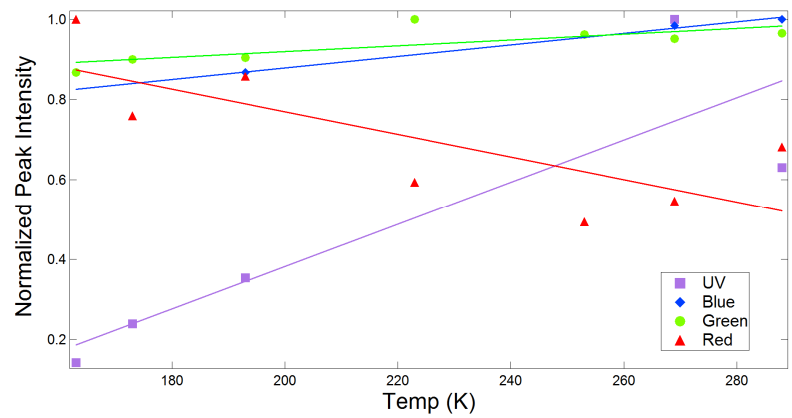

(e)

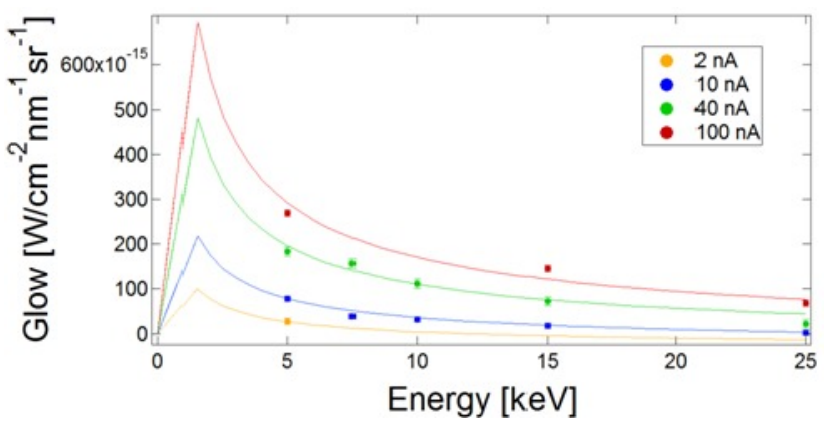

(b)

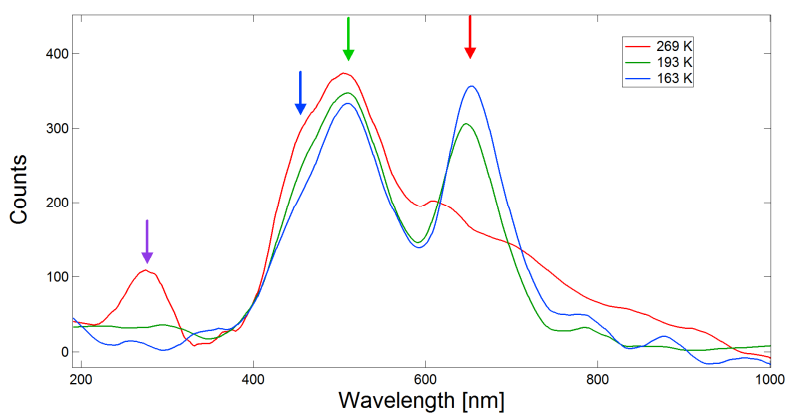

(d)

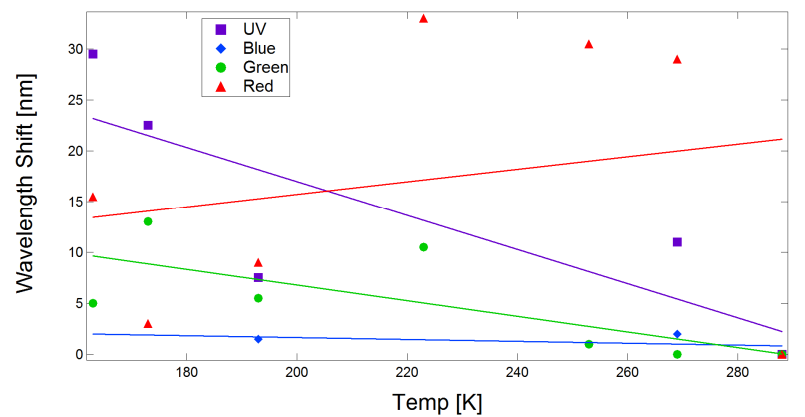

(f)

Figure 4. Optical measurements of luminescent thin film disordered $\mathrm{SiO}_{2}$ samples. (a) Total luminescent radiance versus beam current at fixed incident energy and temperature $(40 \pm 5 \mathrm{~K})$, fit with Eq. (1). (b) Total luminescent radiance versus beam energy at fixed incident flux and temperature $(40 \pm 5 \mathrm{~K})$, fit with Eq. (1). Lower energy data from related experiments 14 are not shown in this plot. (c) Total luminescent radiance versus temperature at fixed incident energy $(5.00 \pm 0.01 \mathrm{keV})$ and incident flux $\left(100 \pm 1 \mathrm{nA} / \mathrm{cm}^{2}\right)$, fit with Eq. (1). (d) Three luminescence UV/VIS spectra at decreasing sample temperature. Four peaks are identified: red $(\sim 645 \mathrm{~nm})$, green $(\sim 500 \mathrm{~nm})$, blue $(\sim 455 \mathrm{~nm})$ and UV $(275 \mathrm{~nm})$. (e) Normalized peak amplitudes as a function of sample temperature of the red $(\sim 645 \mathrm{~nm})$, green $(\sim 500 \mathrm{~nm})$, blue $(\sim 455 \mathrm{~nm})$ and UV ( 275 $\mathrm{nm})$ peaks. These are calculated as the amplitude of each peak (with baseline subtraction) normalized by the largest amplitude for that peak. (f) Peak wavelength shift as a function of sample temperature. Linear fits in (e) and (f) provide guides to the eye of the general trends.

stability of $\pm 1 \mathrm{~K}^{18}$. Though not the focus of this paper, each sample was wired appropriately for collecting electrical data during bombardment as well; these results are reported elsewhere ${ }^{11}$.

\section{RESULTS}

Low intensity cathodeluminescence was observed for disordered $\mathrm{SiO}_{2}$ thin films under low intensity incident electron irradiation over a wide range of temperatures. The thin film $\mathrm{SiO}_{2}$ samples exhibited luminescence (see Fig. 3) readily observable with the naked eye in a darkened room at incident electron powers 10 to 100 times larger than typical of storm conditions in the solar wind, GEO or L2 environments [see Fig. 1(a)]. The intensity scaled with electron beam 
intensity and responded to changes in beam position and profile on rapid time scales, as demonstrated by the image of a defocused and distorted electron beam incident on the sample shown in Fig. 3(d). A clear variation in the intensity and color as a function of temperature was seen with the SLR images of Figs 3(a-c); as temperature increased, the light emission from the samples became bluer and less red. This was confirmed with relative intensity measurements of the Red-Green-Blue bands (390-650 nm total bandpass) of the SLR CCD camera as a function of temperature, as shown in Fig. 3(e).

A more quantitative measure of the cathodoluminescent intensity was obtained from the signal of the CCD video camera (400-900 nm bandpass) averaged over the illuminated area of the sample, as shown in Figs. 4(a-c). These plots are given in terms of calibrated absolute spectral radiance for the beam currents, beam energies, and temperatures noted. Total luminescent radiance increased with increasing beam current at fixed incident energies and temperature, as seen in Fig. 4(a). Total luminescent radiance initially increased with beam energy at fixed incident fluxes and temperature, but decreased above beam energies of $\sim 1.5 \mathrm{keV}$ [see Fig. 4(b)]. Total luminescent radiance in the visible range at fixed incident energy and incident flux increased as temperatures decreased from room temperature to the lowest measured temperatures at $163 \mathrm{~K}$, as shown in Fig. 4 (c).

Dennison et al. have proposed a basic model for the observed electron-induced luminescence phenomenon, based on band theory of highly disordered insulating materials that explains the measurements in Fig. $4{ }^{14}$. The observed luminescence occurs when an incident high energy, charged particle undergoes a series of inelastic collisions exciting valence band electrons into the conduction band. The excited electron rapidly decays to localized (shallow trapped) states, with a mean binding energy $\varepsilon_{S T}$ below the mobility edge. A final electron transition, from the short-lived shallow trap states to longer lived deep trap states is the origin of the emitted photon. The model predicts that the luminescence intensity $I_{\gamma}$ scales with incident current density $J_{b}$, incident beam energy $E_{b}$, temperature $T$, and photon wavelength $\lambda$ as

$$
I_{\gamma}\left(J_{b}, E_{b}, T, \lambda\right) \propto \frac{\dot{D}\left(J_{b}, E_{b}\right)}{\dot{D}+\dot{D}_{s a t}}\left\{\left[e^{-\left(\varepsilon_{S T} / k_{B} T\right)}\right]\left[1-e^{-\left(\varepsilon_{S T} / k_{B} T\right)}\right]\right\}\left\{\left[1-\mathbb{A}_{f}(\lambda)\right]\left[1+\mathbb{R}_{m}(\lambda)\right]\right\},
$$

where the dose rate $\dot{D}$ (absorbed power per unit mass) is given by

$$
\dot{D}\left(J_{b}, E_{b}\right)=\frac{E_{b} J_{b}\left[1-\eta\left(E_{b}\right)\right]}{q_{e} \rho_{m}} \times\left\{\begin{array}{cc}
{[1 / L]} & ; R\left(E_{b}\right)<L \\
{\left[1 / R\left(E_{b}\right)\right]} & ; R\left(E_{b}\right)>L
\end{array}\right.
$$

$q_{e}$ is the electron charge, and $\rho_{m}$ is the mass density of the coating. Fits shown in Fig. 4 were obtained with the overall intensity, $\varepsilon_{S T}$, and $\dot{D}_{s a t}$ as free parameters; only the overall intensity varied from one experiment to the next. A more detailed discussion of the model is given by Dennison et al. ${ }^{14}$. Here we only summarize the dependence of spectral radiance on $J_{b}, E_{b}, T$, and $\lambda$ :

Incident Beam Current. For $\mathrm{SiO}_{2}$ (band gap $\approx 8.9 \mathrm{eV}$ ), excitation from the valence band to conduction band is not thermal, but rather occurs through collisions with the incident high energy electrons. Thus, at low currents, $I_{\gamma}$ is proportional to the incident current, $J_{b}$, through the dose rate. At very high current density, saturation can occur when trap states fill, limiting the number of states electrons can decay into, and leading to $I_{\gamma} \propto\left(\dot{D}+\dot{D}_{s a t}\right)^{-1}$. This saturation behavior is evident in Fig. 4(a). $\dot{D}_{\text {sat }}$ is a material dependant saturation dose rate, found equal to $\sim 10$ $\mathrm{Gy} / \mathrm{s}$ for these disordered $\mathrm{SiO}_{2}$ coatings.

Incident Beam Energy. Below saturation current densities, $I_{\gamma}$ is linearly proportional to $\dot{D}$, given by Eq. (2) as the number areal density of incident electrons per unit time, $\left(J_{b} / q_{e}\right)$, times energy per incident electron, $E_{b}$, divided by the mass into which energy is deposited, $\left(\rho_{m} L \cdot A r e a\right)$. For nonpenetrating radiation-where the energy-dependent penetration depth or range, $R\left(E_{\text {inc }}\right)$, is less than the film thickness $L$-all incident power is absorbed in the coating and $I_{\gamma}$ and $\dot{D}$ are linearly proportional to the incident power density, $\left(J_{i n c} E_{i n c} / q_{e}\right)$. For penetrating radiationwhere $R\left(E_{\text {inc }}\right)>L$-the absorbed power is reduced by a factor of $\left[L / R\left(E_{\text {inc }}\right)\right]^{14}$. At energies up to $\sim 1.5 \mathrm{keV}$ needed to penetrate a $\sim 65 \mathrm{~nm} \mathrm{SiO}{ }_{2}$ coating, $I_{\gamma}$ is linearly proportional to incident flux, energy and power. However, above the coating penetration energy the energy-dependence of $R$ and $\dot{D}$ cause $I_{\gamma}$ to decrease with incident energy (see Fig. 1(b)). This is consistent with the energy-dependant intensity data shown in Fig. 4(b). 
Fig. 1(b) shows $R\left(E_{b}\right)$ for disordered $\mathrm{SiO}_{2}$, along with a corresponding $\dot{D}\left(E_{b}\right)$ curve based on Eq. (2); the extrema in range and dose rate curves occur near $139 \mathrm{eV}$, the mean energy lost by high energy electrons in a single inelastic collision ${ }^{7}$. The maximum energy absorption is $\sim 100 \mathrm{eV}$ for $\mathrm{SiO}_{2}$, falling to $\sim 5 \%$ of the peak absorption at $\sim 20 \mathrm{keV}$. Comparison of Figs. 1(a) and 1(b) shows that the penetration depth in this energy range is $\lesssim 10 \mu \mathrm{m}$ ) and that coatings of any reasonable thickness will have significant power absorbed for electrons of energies up to $\$ 20 \mathrm{keV}$. This also suggests that luminescence may be less of a problem in environments where high energy flux that will pass through most optical coatings dominates, such as GEO.

An energy-dependent correction to the incident flux, $J_{b}\left[1-\eta\left(E_{b}\right)\right]$, is included in Eq. (2) to account for quasielastic backscattered electrons that do not deposit substantial energy; $\eta\left(E_{b}\right)$ is the backscattered electron yield ${ }^{14}$. For the most part, this correction is small and weakly dependant on energy. For biased samples, or when excess charge is stored in the trap states, a surface voltage $V_{s}$ results and $E_{\text {inc }}$ is replaced everywhere in Eqs. (1) and (2) by the landing energy, $\left(E_{b}-q_{e} V_{s}\right)$. Thus, glow intensity may diminish appreciably for $\mathrm{kV}$ surface potentials often observed on GEO satellites in eclipse.

Temperature. The thermal dependence of luminescence in equilibrium is proportional to the number of electrons in the conduction band that can fall into the shallow traps $\left(\propto\left[e^{-\left(\varepsilon_{S T} / k_{B} T\right)}\right]\right)$ and to the fraction of electrons that are retained in the shallow traps and not thermally excited into the conduction band $\left(\propto\left[1-e^{-\left(\varepsilon_{S T} / k_{B} T\right)}\right]\right)$. Thus, $I_{\gamma} \propto\left\{\left[e^{-\left(\varepsilon_{S T} / k_{B} T\right)}\right]\left[1-e^{-\left(\varepsilon_{S T} / k_{B} T\right)}\right]\right\}$. At higher temperatures where $k_{B} T \gg \varepsilon_{S T}$, the thermal energy exceeds the mean energy depth of the shallow traps below the conduction band, $\varepsilon_{S T}$, and $I_{\gamma} \propto\left(\varepsilon_{S T_{Q}} / k_{B} T\right)$. This is consistent with the results in Fig. 4(c), with $\varepsilon_{S T} \approx 41 \mathrm{meV}$.

Wavelength. The final term in curly brackets in Eq. (1) accounts approximately for photons propagation through the coating. The terms shown in Eq. (1) are first order, and assume the optical absorption of the coating materials, $\mathbb{A}_{f}(\lambda) \ll 1$ for propagation through the full film. For coating materials with very high optical absorption, $I_{\gamma}$ is limited to emission from the near-surface region from which photons can escape and is approximately independent of electron energy. $\mathbb{R}_{m}(\lambda)$ is the reflectivity of the metallic layer. For highly reflective materials like Ag, $\mathbb{R}_{m} \approx 1$ at all $\lambda$ considered. For materials like $\mathrm{Au}$, the glow spectrum is enhanced at longer $\lambda$, due to greater absorptivity of $\mathrm{Au}$ at shorter $\lambda$. For less reflective materials, $\mathbb{R}_{m} \ll 1$ at all $\lambda$, hence photons reflected from the metallic layer do not substantially increase the luminescence.

Luminescence from disordered $\mathrm{SiO}_{2}$ will be most significant in instruments operating primarily in the visible portion of the spectrum. The measured relative cathodoluminescence UV/VIS/NIR spectra of $\mathrm{SiO}_{2}$ [see Fig. 4(d)] are most pronounced in the visible range, extending from $\sim 250 \mathrm{~nm}$ to $\sim 900 \mathrm{~nm}$. The spectra are consistent with the number, relative intensity and wavelengths of peaks observed in previous room temperature studies for similar disordered $\mathrm{SiO}_{2}$ samples ${ }^{10,19-21}$. Three peaks are seen, centered at approximately $275 \mathrm{~nm}, 500 \mathrm{~nm}$ and $645 \mathrm{~nm}$; an additional shoulder is observed at $\sim 455 \mathrm{~nm}$. Sahl et al. identified the peak at $645 \mathrm{~nm}$ as a nonbridging oxygen hole center in the structure ${ }^{19}$. The peaks at $275 \mathrm{~nm}$ and $500 \mathrm{~nm}$ were attributed to an oxygen deficient center by Trukhin et $l^{20}$.

The variation in spectral intensity with temperature is shown for three representative temperatures in Fig. 4(d). The observed intensity decrease with increasing temperature in the red peak near $645 \mathrm{~nm}$ and the increase in the blue and UV peaks near $455 \mathrm{~nm}$ and $275 \mathrm{~nm}$, respectively, are consistent with the qualitative SLR observations seen in Fig. 3. A shift of luminescent peaks to shorter wavelengths with increasing temperature was observed for all but the red peak [see Fig. 1(f)]. A model with multiple deep trap bands is necessary to explain the four identifiable UV/VIS peaks observed in the spectra, and has been used to qualitatively explain the observed temperature dependence of the peak position and intensity ${ }^{14}$.

\section{ADDITIONAL CONSIDERATIONS}

Detection of extremely faint signals (distant objects) observed by space-based observatories can be limited by background light levels from a variety of sources. These can include contributions from astronomical sources within and outside our solar system (zodiacal and galactic background, respectively), contributions from the observatory itself (i.e., thermal self emission in the infrared), and scattered light from the combination of astronomical sources and observatory properties. Ambient operating temperature of $\sim 40 \mathrm{~K}$ reduces thermal background radiance below the 
zodiacal background, except for mid-IR wavelengths $>10 \mu \mathrm{m}{ }^{17}$. Cathodoluminescence from electron impact onto coatings or optical components, constitutes yet another possible source of background light. Ferguson et al. compare the cathodoluminescence radiance to solar radiance, Earthshine radiance, equilibrium thermal emittance, and zodiacal background radiance at LEO and GEO orbits ${ }^{22}$. Further from the Earth, for Lissajous orbits about the Earth-Sun L2 Lagrange libration point ${ }^{1}$, contributions from the Earthshine and moonshine and from bright point sources near the field of view were estimated to be at least an order of magnitude less than the limit from the zodiacal sky ${ }^{17,23}$. Thus, a maximum stray light condition is often set by the zodiacal sky background ${ }^{1,16,22}$. As a reference point, the intensity of the zodiacal background at $1 \mathrm{AU}$ near the south ecliptic pole is $8 \cdot 10^{-14} \mathrm{~W} \mathrm{~cm}^{-2} \mathrm{~nm}^{-1} \mathrm{sr}^{-1}$ at $500 \mathrm{~nm}, 3 \cdot 10^{-14} \mathrm{~W} \mathrm{~cm}^{-2} \mathrm{~nm}^{-1}$ $\mathrm{sr}^{-1}$ at $1000 \mathrm{~nm}$, and $6 \cdot 10^{-15} \mathrm{~W} \mathrm{~cm}^{-2} \mathrm{~nm}^{-1} \mathrm{sr}^{-1}$ at $2000 \mathrm{~nm}^{16}$.

To determine the magnitude of cathodoluminescence for a specific space-based observatory situation, to compare with these background sources, requires knowledge of:

(i) The observatory electron environment and specifically the electron flux spectrum. Representative spectra for several common environments shown in Fig. 1 (a) provide typical ranges, but fluxes for each observatory will depend on the particular environments that are encountered throughout the mission as well as temporal variations (e.g., phase of solar cycle, space weather events, etc.). This may require consideration of a range of fluxes and worst case situations. In almost all space environmental conditions, the incident flux is low enough for the cathodoluminescent intensity to be linearly proportional to the absorbed dose rate.

(ii) The degree of baffling or shielding of electrons onto potentially luminescent optics. An open architecture and minimal shielding, often required for large observatories due to size and mass constraints, permits stray light to enter the optical path of the telescope and also exposes optical elements and surrounding support structures to greater environmental electron fluxes. Some optical surfaces may have significant baffling against electron flux, either as a result of dedicated stray light baffling or by their location within a system with effective baffling due to nearby structure. The effect of such baffling can depend on the orientation and directionality of the electron flux as well. It also can depend on incident energy-dependant attenuation of the environmental flux by shielding. For example, incident electrons will lose approximately $20 \mathrm{keV}$ in penetrating $\sim 8-25 \mu \mathrm{m}$ of $\mathrm{SiO}_{2}$ or polymeric shielding [see Fig. 1(b)], but electrons above $\sim 20 \mathrm{keV}$ could still produce cathodoluminescence on interior surfaces. Thus, for a solar wind environment, a 1 mil thick blanket would reduce interior luminescence by more than 3 orders of magnitude, but the sample blanket would have little effect in a GEO environment [see Fig. 1(a)].

(iii) The effectiveness of the optical system in transmitting the generated cathodoluminescence from the impacted optics to instrument focal planes. This depends, for example, on the juxtaposition of the luminescent elements and the location of stops along the optical path. Because the $\mathrm{SiO}_{2}$ optical coatings studied here are often found on lenses and windows directly along the optical path, the effects can be much higher than for stray light which could be generated by luminescent support structures or shields outside or at the periphery of the optical path.

(iv) The thickness of the luminescent coating. The total luminescent intensity has a critical dependence on the film thickness as compared with the incident electron energy- dependent penetration depth; this ratio determines the fraction of power deposited in the coating (see Fig. 4(b) and Eq. 2) and the resulting luminescent intensity. This analysis is more complicated for a distribution of energies in a given environmental flux that spans both penetrating and nonpentrating radiation. For example, for $\sim 60 \mathrm{~nm}$ to $\sim 200 \mathrm{~nm}$ thick $\mathrm{SiO}_{2}$ coatings studied here, the vast majority of electron flux for the solar wind environment is below $\sim 1 \mathrm{keV}$ and hence nonpenetrating, while the majority of electron flux in the GEO environment is above $\sim 20 \mathrm{keV}$ and hence penetrates the $\mathrm{SiO}_{2}$ coatings. Other environments shown in Fig. 1(a) have a distribution of both penetrating and nonpenetrating radiation.

(v) The temperature of the luminescent coating. As shown here, lower operating temperature for long wavelength sensitive observatories will lead to higher luminescence, down to $\sim 90 \mathrm{~K}$; below this temperature luminescence decreases slowly. While the shape of the luminescence versus temperature curve should be similar for different materials, the temperature of the inflection point depends on the energy distribution of the shallow defect states which can vary substantially for otherwise similar materials. See Fig. 4(d) and Eq. (1).

(vi) The composition of the luminescent coating. To a modest extent, the luminescent spectrum and overall intensity will change with the exact composition and atomic scale structure of the luminescent coating. We present here measurements for disordered $\mathrm{SiO}_{2} / \mathrm{SiO}_{\mathrm{x}}$ coatings, but expect that similar effects are likely for other 
common protective coatings - e.g., other forms of disordered $\mathrm{SiO}_{2}, \mathrm{Al}_{2} \mathrm{O}_{3}$, and $\mathrm{MgF}$ coatings - due to the similarities of their band structure. A related issue is the effect of coating contamination or surface modification on coating reflectivity and luminescence ${ }^{24}$. These effects were not addressed quantitatively in this study; however, Lightsey et al. ${ }^{1}$ provide an excellent discussion of the effects of contamination on optical elements in relation to stray light background that is applicable to coating contamination.

It is important to be aware as well of the close coupling of cathodoluminescence with spacecraft charging effects. Charging and electrostatic discharges are caused by the same electron plasma fluxes that induce cathodoluminescence, and share many of the same dependence on incident energy, incident flux, and sample temperature ${ }^{11,25}$. Another related consequence of arcing is the possibility of sputtering of optical coating or reflective layers due to discharge ${ }^{11}$. This can be particularly problematic if the reflective coatings are not electrically grounded, which can lead to enhanced arcing. The type and size of damage sites from arc-induced sputtering and further surface contamination from arc-induced ejecta, and their effects on optical performance, can be compared to damage from micrometeroid impacts addressed by Lightsey ${ }^{1}$ and others.

Cathodoluminescence can also be potentially useful. One such possibility is to provide the capability for remote monitoring of the evolution of satellite surfaces, using natural space plasma as an excitation source. Alternately, materials that have particularly high intensity could be mounted on spacecraft to provide remote monitoring of such things as contamination and spacecraft potential. Ferguson et al. reviewed these possibilities ${ }^{22}$. They concluded that it was unlikely that direct observations of such luminescence could be made from Earth-based telescopes or even of GEO satellites from LEO observatories; however, the possibility of observations of these phenomena from co-orbiting LEO or GEO satellites seemed feasible.

\section{CONCLUSION}

Disordered, thin film $\mathrm{SiO}_{2} / \mathrm{SiO}_{\mathrm{x}}$ coatings, commonly used as protective mirror coatings, exhibit cathodoluminescence under electron-beam bombardment. Our measurements indicate that this emission arises primarily from four distinct bands observed in UV/VIS/NIR $(275-1000 \mathrm{~nm})$. The intensity increases and reddens at lower temperatures. We also measured the radiance of this glow as a function of electron energy and beam current. A simple model was proposed that describes the dependence of this cathodoluminescence on irradiation time, incident flux and energy, sample thickness, and temperature. Other factors necessary for the estimation of the magnitude and effect of this potential source of background light for a particular mission include: the electron environment and variability, considerations of baffling of the impacted elements, transmission of this luminescent light to instrument focal planes, thickness of the coatings used on particular elements, and the temperatures of the coated elements. The information provided in this study, coupled with these specific mission specifications, is sufficient to determine the risks posed to the performance of spaced-based observatories from space plasma environmentally-induced cathodoluminescence.

In efforts to explore further into the farthest reaches of our universe, astronomical observatories continue to expand in size and complexity and to be located in more exotic and demanding environments. As future, more complex and sensitive missions push the envelope into more extreme environments, the fundamental understanding of luminescent dependences of spacecraft materials on time, temperature, flux, energy, and material structure from this work, provides a key factor for estimating and minimizing the deleterious effects of this radiation.

\section{ACKNOWLEDGMENTS}

We gratefully acknowledge contributions to instrumentation and experimental efforts from Robert Johnson, Jennifer Albretsen Roth and Doug Ball of the USU Materials Physics Group, help with optical calibrations from James Peterson of the USU Space Dynamics Laboratory, and Michael Taylor for the use of infrared and CCD video cameras. We also acknowledge the many useful discussions about this work from contributors to the JWST Electrical Systems Working Group conference calls. This work was supported by a project through the NASA Goddard Space Flight Center and a NASA Space Technology Research Fellowship (Jensen). 


\section{REFERENCES}

[1] Lightsey, P. A., Atkinson, C., Clampin, M., and Feinberg, L. D., "James Webb Space Telescope: large deployable cryogenic telescope in space", Opt. Eng. 51(1), 011003 (2012).

[2] Dennison, J. R., Jensen, A. E., Dekany, J. Wilson, G., Bowers, C. W. and Meloy, R., "Diverse Electron-induced Optical Emissions from Space Observatory Materials at Low Temperatures," Proc. SPIE, San Diego, CA, Aug. (2013).

[3] Hastings, D. and Garrett H., [Spacecraft-Environment Interactions], New York, NY: Cambridge Press, (1996).

[4] Minow, J., private communications, (2010).

[5] Minow, J., Parker, L. N., Alstaff, R. L., Blackwell Jr., W. C., Dickmann, A., "Radiation and Internal Charging Environments for Thin Dielectrics in Interplanetary Space," Proc. $9^{\text {th }}$ Spacecraft Charging Techn. Conf., (Epochal Tsukuba, Tsukuba, Japan, April (2005).

[6] Minow, J., Blackwell, W. and Diekmann, A., "Plasma Environment and Models for L2," AIAA Paper No. AIAA2004-1079, 42 $2^{\text {nd }}$ Aerospace Sciences Meeting and Exhibit, Jan. (2004).

[7] Wilson, G. and Dennison, J. R., "Approximation of Range in Materials as a Function of Incident Electron Energy," IEEE Trans. on Plasma Sci., 40(2), 305-310 (2012).

[8] Leach, R., and M. Alexander, "Failures and anomalies attributed to spacecraft charging," NASA STI/Recon Technical Report N 96, 11547 (1995).

[9] Griseri, V., Dissado, L. A., Fothergill, J. C., Laurent, C. and Teyssedre, G., "Photoluminescence, recombination induced luminescence and electroluminescence in epoxy resin," J. Phys. D: Appl. Phys, 34, 2534-2540 (2001).

[10]Fitting, H. -J., Barfels, T., von Czarnowski, A. and Trukhin, A. N., "Electron Beam Induced Optical and Electronical Properties of $\mathrm{SiO}_{2}$," Materials Science and Engineering, B71, 109-114 (2000).

[11] Wilson, G., Evans, A., Dekany, J. and Dennison, J. R., "Charging Effects of Multilayered Dielectric Spacecraft Materials: Surface Voltage, Discharge and Arcing,” IEEE Trans. on Plasma Sci., (2013), in press.

[12] Jensen, A. E., Wilson, G., Dekany, J., Sim, A. M. and Dennison, J. R, "Low Temperature Cathodoluminescence of Space Observatory Materials," IEEE Trans. on Plasma Sci., (2013), in press.

[13] Jensen, A. E., Dennison, J. R., Wilson, G. and Dekany, J., "Nanodielectric Properties of High Conductivity CarbonLoaded Polyimide under Electron-Beam Irradiation," Proc. ICSD, (2013).

[14]Dennison, J. R., Evans, A., Wilson, G., Dekany, J., Bowers C. W., and Meloy, R., "Electron Beam Induced Luminescence of $\mathrm{SiO}_{2}$ Optical Coatings," Proc. IEEE-CEIDP (2012).

[15]Dennison, J. R., "Characterization of Electrical Materials Properties Related to Spacecraft Charging," Radiation Capabilities for the Europa Jupiter System Missions Instrument Workshop, Johns Hopkins Applied Physics Laboratory, Laurel, MD, July (2009).

[16] Leinert, C. et al., "The 1997 Reference of Diffuse Night Sky Brightness," Astron. Astrophys. Suppl. Ser., 127, 1 (1998).

[17]Lightsey, P.A., and Wei, Z., "James Webb Space Telescope observatory stray light performance", Proc. SPIE6265, Space Telescopes and Instrumentation I: Optical, Infrared, and Millimeter, 62650S, June (2006).

[18] Dekany, J., Johnson, R. H., Wilson, G., Evans, A. and Dennison, J. R., "Ultrahigh Vacuum Cryostat System for Extended Low Temperature Space Environment Testing," IEEE Trans. on Plasma Sci., (2013), in press.

[19] Salh, R., von Czarnowski, A., Zamoryanskaya, M. V., Kolesnikova E. V. and Fitting, H. -J., "Cathodoluminescence of $\mathrm{SiO}_{\mathrm{x}}$ Under-stoichiometric Silica Layers," Phys. Stat. Sol., 203, 2049-2057 (2006).

[20] Trukhin, A. N., Goldberg, M., Jansons, J., Fitting, H. -J. and Tale, I. A., "Silicon Dioxide Thin Film Luminescence in Comparison with Bulk Silica," J. Non-Crystalline Solids, 223, 114-122 (1998).

[21] Fitting, H. -J., Barfels, T., Trukhin, A. N. and Schmidt, B., "Cathodoluminescence of crystalline and amorphous $\mathrm{SiO}_{2}$ and $\mathrm{GeO}_{2}$," J. Non-Crystalline Solids, 279, 51-59 (2000).

[22] Ferguson, D. C., Krezan, J. -M., Barton, D. A., Dennison, J. R. and Gregory, S., "On the Feasibility of Detecting Spacecraft Charging and Arcing by Remote Sensing," Paper Number, AIAA-2013-, $5^{\text {th }}$ AIAA Atmospheric and Space Environments Conference, San Diego, CA, June (2013).

[23] Wei, Z. and Lightsey, P.A., "Stray light from galactic sky and zodiacal light for JWST", Proc. SPIE 6265, Space Telescopes and Instrumentation I: Optical, Infrared, and Millimeter, 62653C, June (2006).

[24] Evans, A. and Dennison, J. R., "The Effects of Surface Modification on Spacecraft Charging Parameters," IEEE Trans. on Plasma Sci., 40(2), 291-297 (2012).

[25] Dennison, J. R., Wilson, G., Hoffmann, R. and Evans, A., "Comparison of Radiation Induced Conductivities at Low Temperature,” Bull. Am. Phys. Soc. 57(1), APS March Meeting, Baltimore, MD, March (2013). 